\title{
Age-related macular degeneration affects the optic radiation white matter projecting to locations of retinal damage
}

\author{
Shoyo Yoshimine ${ }^{1} \cdot$ Shumpei Ogawa ${ }^{1,2} \cdot$ Hiroshi Horiguchi ${ }^{1} \cdot$ Masahiko Terao $^{3} \cdot$ Atsushi Miyazaki $^{4}$. \\ Kenji Matsumoto ${ }^{5} \cdot$ Hiroshi Tsuneoka $^{1} \cdot$ Tadashi Nakano $^{1} \cdot$ Yoichiro Masuda $^{1} \cdot$ Franco Pestilli $^{6,7,8,9,10,11}$ [D
}

Received: 6 September 2017 / Accepted: 17 June 2018 / Published online: 27 June 2018

(c) The Author(s) 2018

\begin{abstract}
We investigated the impact of age-related macular degeneration (AMD) on visual acuity and the visual white matter. We combined an adaptive cortical atlas and diffusion-weighted magnetic resonance imaging (dMRI) and tractography to separate optic radiation (OR) projections to different retinal eccentricities in human primary visual cortex. We exploited the known anatomical organization of the OR and clinically relevant data to segment the OR into three primary components projecting to fovea, mid- and far-periphery. We measured white matter tissue properties-fractional anisotropy, linearity, planarity, sphericity-along the aforementioned three components of the optic radiation to compare AMD patients and controls. We found differences in white matter properties specific to OR white matter fascicles projecting to primary visual cortex locations corresponding to the location of retinal damage (fovea). Additionally, we show that the magnitude of white matter properties in AMD patients' correlates with visual acuity. In sum, we demonstrate a specific relation between visual loss, anatomical location of retinal damage and white matter damage in AMD patients. Importantly, we demonstrate that these changes are so profound that can be detected using magnetic resonance imaging data with clinical resolution. The conserved mapping between retinal and white matter damage suggests that retinal neurodegeneration might be a primary cause of white matter degeneration in AMD patients. The results highlight the impact of eye disease on brain tissue, a process that may become an important target to monitor during the course of treatment.
\end{abstract}

Keywords White matter $\cdot$ Diffusion $\cdot$ MRI $\cdot$ Age-related macular degeneration $\cdot$ Tractography

Electronic supplementary material The online version of this article (https://doi.org/10.1007/s00429-018-1702-5) contains supplementary material, which is available to authorized users.

Shoyo Yoshimine

s.yoshimine@jikei.ac.jp

$\triangle$ Franco Pestilli

franpest@indiana.edu

1 Department of Ophthalmology, The Jikei University School of Medicine, 3-25-8 Nishi-Shinbashi, Minato-ku, Tokyo 105-8461, Japan

2 Department of Ophthalmology, Atsugi City Hospital, Kanagawa, Japan

3 Research Institute for Time Studies, Yamaguchi University, Yamaguchi, Japan

4 Tamagawa University Research Institute, Machida, Tokyo, Japan

5 Tamagawa University Brain Science Institute, Machida, Tokyo, Japan
6 Department of Psychological and Brain Sciences, Indiana Network Science Institute, Indiana University, Bloomington, IN 47405, USA

7 Department of Computer Science, Indiana University, Bloomington, USA

8 Department of Intelligent Systems Engineering, Indiana University, Bloomington, USA

9 Program in Neuroscience, Indiana University, Bloomington, USA

10 Program in Cognitive Science, Indiana University, Bloomington, USA

11 School of Optometry, Indiana University, Bloomington, USA 


\section{Introduction}

Human aging can be associated with degeneration of both or either the retinal pigment epithelium and photoreceptor cells. Such degeneration often starts at the center of the retina in the Macula, a clinical syndrome referred to as age-related macular degeneration (AMD). AMD often results in irreversible loss of visual function. By affecting more than $25 \%$ of individuals older than 75 years (Ratnapriya and Chew 2013), AMD has been prospected to become a disease of global impact with a total number of individuals affected by 2020 reaching 196 millions worldwide (Wong et al. 2014).

Modern technologies for mapping the human brain allow investigators to measure both micro- and macroscopic properties of the white matter tissue organization and composition in vivo; these methods have the potential to diagnose and track disease progression across the lifespan. Diffusion-weighted magnetic resonance imaging (dMRI) and computational tractography are two methods that, when combined, can map the macroscopic anatomical organization as well as the microscopic tissue properties of the human white matter (Jbabdi et al. 2015; Wandell 2016; Rokem et al. 2017).

Measurements using dMRI have shown biological changes in white matter associated with neurodegeneration. For example, the human white matter is affected during normal aging as well as in age-related diseases, such as Alzheimer's or progressive cognitive degeneration (Mayda et al. 2010; Wang et al. 2010, 2012; Yeatman et al. 2014; Thomason and Thompson 2011). The visual white matter pathways have been also shown to be affected by visual and eye disease, for example, developmental prosopagnosia (Gomez et al. 2015; Thomas et al. 2008), amblyopia (Allen et al. 2015, 2018; Duan et al. 2015), and retinal ganglion cell damage (Ogawa et al. 2014) have all been reported to affect properties of the human white matter, please see Ariel Rokem et al. 2017; Millington et al. 2014 for reviews. Evidence from in vivo studies suggest that macular degeneration also affects volume of the human primary visual cortex and the visual pathways (Prins et al. 2016a, b; Hernowo et al. 2014; Malania et al. 2017). Yet to date, the complete set of mechanisms by which AMD has long-term effects on the human brain and behavior are not well understood. Importantly, the degree to which the changes in visual white matter can inform AMD diagnosis-i.e., classify individuals as AMD patient or within the normal aging population-has not been established. We address both these points.

We estimated the properties of the visual white matter using multiple models of tissue microstructure-fractional anisotropy (Basser and Pierpaoli 1998), planarity, and sphericity (Carl-Fredrik Westin et al. 1997, 2002). Critically, we show changes in white matter tissue properties within the optic radiation that are specific to fascicles projecting from central, foveal locations, those with retinal damage. To do so, we performed an advanced, anatomically precise characterization of the relationship between the locus of retinal damage in AMD (fovea) and the white matter fascicles within the optic radiation projecting to locations of primary visual cortex that represent different eccentricities - fovea, parafoveal and peripheral regions combining anatomical and advanced computational methods (Benson et al. 2012, 2014). This fine grade mapping between the retinotopic location of damage and white matter goes beyond previous reports (Prins et al. 2016a, b; Hernowo et al. 2014; Malania et al. 2017). Furthermore, we demonstrate that changes in the microstructural properties of the OR correlate with AMD patients' visual acuity, indicating a relationship between the tissue properties of the OR and the long-term loss in visual function that characterizes AMD. These results show a conserved mapping between the location of retinal damage and white matter fascicles, suggesting that retinal neurodegeneration might cause long-term changes in the visual white matter of the OR in AMD patients.

\section{Methods}

This research was approved by the ethical committees of the Jikei University School of Medicine, Tamagawa University, and Atsugi City Hospital. All participants provided written informed consent to participate in the project. All procedures conformed to the Declaration of Helsinki.

Two experienced ophthalmologists (one was the first author) diagnosed AMD at the Jikei University School of Medicine or at the Atsugi City Hospital. All subjects were given an ophthalmological examination, including best-corrected visual acuity, intraocular pressure, slit lamp microscopy, fundus examination, and optical coherence tomography (OCT) measurements. To do this, we first measured visual acuity, which is a perception of minimum angle of resolution, in patients and control subjects using a standard Landolt-C stimulus. Subjects performed a four-alternativeforced-choice task by indicating the side toward which the " $C$ " of the Landolt stimulus faced (top, bottom, left or right). For statistical analysis, visual acuity was converted to a logarithmic minimum angle of resolution ( $\log M A R)$.

Eight patients suffered from AMD (seven males and one female; mean age 74.8 years, range $62-84$ years). Six of them had bilateral AMD. The others had unilateral damage. All AMD subjects were undergoing anti-vascular endothelial growth factor therapy (anti-VEGF) (Brown et al. 2006; Heier et al. 2012; Krüger et al. 2013). Twelve 
Table 1 Biographic and biometric measurements of the AMD patients and control participants

\begin{tabular}{|c|c|c|c|c|c|c|c|c|c|c|}
\hline \multirow[t]{2}{*}{ Patient } & \multirow[t]{2}{*}{ Sex } & \multirow[t]{2}{*}{ Affected eye } & \multirow[t]{2}{*}{ Age } & \multicolumn{2}{|c|}{$\log$ MAR VA } & \multicolumn{2}{|l|}{ Macular evaluation } & \multirow[t]{2}{*}{ Control } & \multirow[t]{2}{*}{ Sex } & \multirow[t]{2}{*}{ Age } \\
\hline & & & & $\mathrm{R}$ & $\mathrm{L}$ & $\mathrm{R}$ & $\mathrm{L}$ & & & \\
\hline 1 & $\mathrm{M}$ & B & 62 & -0.08 & 0.00 & PED & PED & 1 & M & 74 \\
\hline 2 & M & $\mathrm{L}$ & 72 & -0.19 & 0.05 & Normal & PED & 2 & $\mathrm{~F}$ & 68 \\
\hline 3 & M & B & 68 & 0.82 & 0.82 & Atrophic & Atrophic & 3 & M & 63 \\
\hline 4 & $\mathrm{~F}$ & B & 72 & 1.22 & 1.00 & Atrophic & Atrophic & 4 & M & 61 \\
\hline 5 & M & $\mathrm{B}$ & 84 & 0.70 & 1.05 & Atrophic & Atrophic & 5 & $\mathrm{~F}$ & 71 \\
\hline 6 & M & B & 79 & 1.22 & 0.70 & Atrophic & Atrophic & 6 & M & 66 \\
\hline 7 & M & B & 82 & 1.10 & 1.00 & Atrophic + edema & Atrophic & 7 & $\mathrm{~F}$ & 61 \\
\hline \multirow[t]{5}{*}{8} & M & $\mathrm{R}$ & 79 & 0.10 & -0.18 & SRD & Normal & 8 & M & 62 \\
\hline & & & & & & & & 9 & $\mathrm{~F}$ & 70 \\
\hline & & & & & & & & 10 & $\mathrm{~F}$ & 65 \\
\hline & & & & & & & & 11 & F & 64 \\
\hline & & & & & & & & 12 & $\mathrm{~F}$ & 59 \\
\hline
\end{tabular}

The $\log M A R V A$ measurements indicate the minimum angle of resolution visual acuity in log units $P E D$ pigment epithelial detachment, $S R D$ serous retinal detachment

The first eight columns report information about patients; last three columns control subjects. Letters $\mathrm{B}, \mathrm{R}$ and $\mathrm{L}$ stand for bilateral, right and left eye, respectively control subjects (six males; mean age 65.3 years, range 59-74 years) with normal, or corrected to normal, visual acuity. None of the patients or controls had a history of brain disease. See Table 1 for additional details about the patients' and controls' biographical and biometric information.

Structural evaluation of the retina was performed using optical coherence tomography (Cirrus HD-OCT; Carl Zeiss Meditec, Dublin, CA, USA). A typical fundus and optical coherence tomography (OCT) image in patient with AMD is shown in Fig. 1. The OCT data distinguish between patients with other different diseases.

\section{Visual field measurement}

Visual field measurements were acquired in six patients using standard Goldmann perimetry. Two patients did not consent to receive the perimetry test. The distribution of visual sensitivity across the visual field differed across subjects. We used kinetic targets to define the absolute scotoma as the region in which subjects could not detect the highest contrast and largest size stimuli V/4e; $64 \mathrm{~mm}^{2}$ (visual angle $1.72^{\circ}$ diameter), $318 \mathrm{~cd} / \mathrm{m}^{2}$. Relative scotoma was defined as the region in which subject could not detect the stimuli I/2e and I/3e; $0.25 \mathrm{~mm}^{2}$ (visual angle $0.11^{\circ}$ ). Five eyes had relative scotoma and five eyes had absolute scotoma. Two eyes were normal (see Fig. 1c, for example, measurements). The position of the (absolute or relative) scotoma was recorded in all patients closest to macula (center of the visual field).

\section{Magnetic resonance image data acquisition}

Magnetic resonance imaging (MRI) data acquisition and preprocessing methods used in this study were identical to those developed for (Ogawa et al. 2014). Below, we briefly describe the major details of MRI measurements and analyses.

All data were acquired at the Brain Science Institute of Tamagawa University, Tokyo, Japan. High-resolution T1-weighted anatomical images were acquired for each subject using a three-dimensional spoiled-gradient recalled acquisition (SPGR; $1 \times 1 \times 1 \mathrm{~mm}$ voxel size; scan duration 9 min, $18 \mathrm{~s}$ ) with sagittal plane acquisition on 3T Siemens MAGNETOM Trio Tim scanner (Siemens, Erlangen, Germany) using a 12-channel head coil.

Diffusion-weighted magnetic resonance images were acquired using two repetitions of a single-shot spin-echo planar imaging sequence (93 ms echo time (TE); 7.5 s repetition time (TR); $230 \mathrm{~mm}$ field of view; $230 \times 56$ matrix size; $\pm 1562 \mathrm{~Hz} / \mathrm{Px}$ bandwidth; $317 \mathrm{~s}$ duration). We acquired 56 axial, 1.8-mm-thick slices (no gap) for two $b$ values, $\mathrm{b}=0$ and $b=1000 \mathrm{~s} / \mathrm{mm}^{2}$. The $b=1000$ data were obtained by applying gradients along 12 unique diffusion directions.

\section{Data preprocessing}

Anatomical images were aligned to the anterior commissure-posterior commissure (AC-PC) plane using a $6^{\circ}$ rigidbody transformation. Diffusion-weighted images were corrected for eddy currents, motion compensated, and aligned to the high-resolution anatomical images using a 14-parameter 


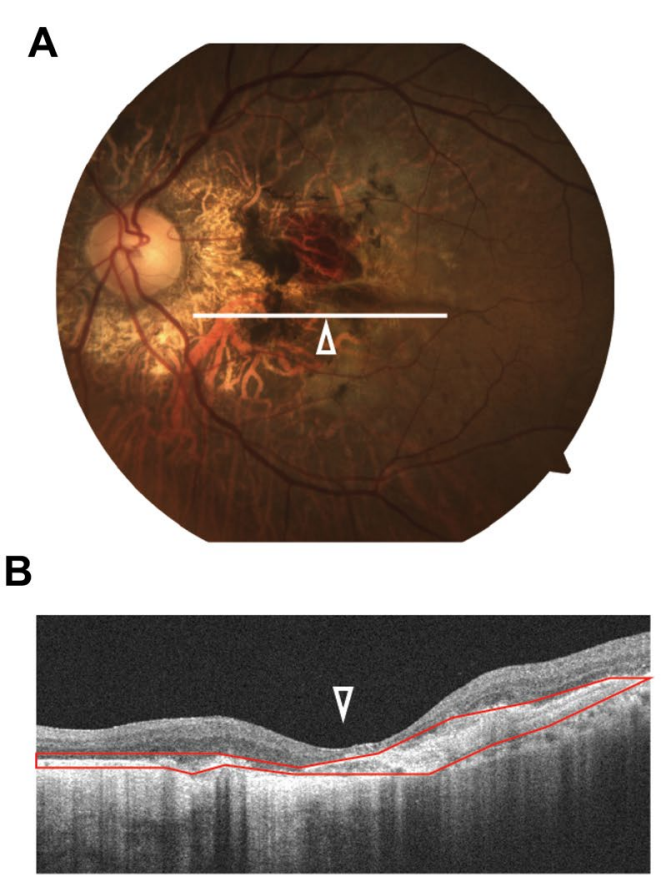

Fig. 1 Measurements of retinal damage. Measurements are reported for a single left eye in a-c. a Example: fundus (retinal image). Lefthand panel. The white horizontal line indicates the approximate location of the optical coherence tomography measurement (OCT; b). The white triangle marks the center of the fovea. Right-hand panel. Fundus and OCT image for one example control subject. b OCT image. Section of the retina through the white line in a. The red region indicates the retinal area with AMD-related tissue degeneration. The structure of outer retina layer is almost completely degen-

constrained nonlinear coregistration. All diffusion-weighted images were resampled to $2 \mathrm{~mm}$ isotropic resolution using trilinear interpolation (Ashburner and John 2009, 2012). An eddy-current intensity correction was applied to the diffusion-weighted images at the resampling stage. Diffusionweighting gradient directions were reoriented by applying the same transformation used on the diffusion-weighted images.

\section{Diffusion tensor model}

Fractional anisotropy and mean, radial and axial diffusivity were estimated by fitting a diffusion tensor model to each voxel signal (Basser and Pierpaoli 1998). Fractional anisotropy (FA) was defined as the normalized standard deviation of the three eigenvalues of the diffusion tensor and indicates the degree to which the diffusion ellipsoid is anisotropic (i.e., one or two eigenvalues are larger than the mean of all three eigenvalues). Mean diffusivity (MD) was defined as the mean of the three eigenvalues. Axial diffusivity (AD) was defined as the apparent diffusion

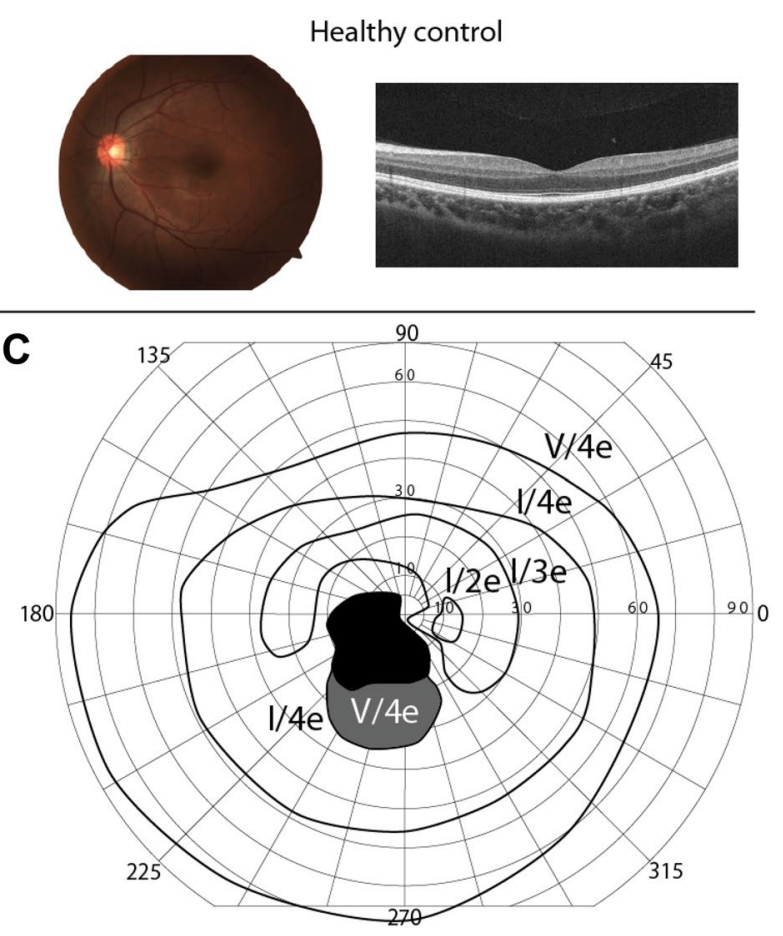

erated. The inner/outer segment line is invisible, indicating photoreceptor damage. White triangle indicates the fovea as in a. c Visual field, as measured by Goldmann perimetry. Black depicts regions of absolute scotoma. The participant could not detect even the largest and brightest stimuli in Goldmann perimetry set. Paracentral gray indicates retinal areas where the patient could detect only the largest and brightest stimulus (V/4e) in the Goldmann perimetry set. Other regions mark locations with either limited damage or no damage

coefficient measured along the principal axis of the tensor model in each voxel. Radial diffusivity (RD) was defined as the average of the diffusivity in the two minor axes of the tensor model. All image analysis software is open source and published at https://github.com/vistalab/vista soft (Ariel Rokem et al. 2015; Pestilli et al. 2014; Dougherty et al. 2007).

\section{Brain segmentation and regions of interest definition}

The brain cortical surface and optic chiasm were reconstructed and segmented using FreeSurfer(Bruce Fischl 2012); version 5.1.0; (http://surfer.nmr.mgh.harvard.edu). We used a probabilistic anatomical atlas to estimate the location of primary visual cortex (Bruce Fischl 2012; Fischl et al. 2007; Hinds et al. 2008). The approximate location of the lateral geniculate nucleus (LGN) was estimated manually on the high-resolution anatomical image (Ogawa et al. 2014; Allen et al. 2015; Millington et al. 2014). 


\section{Anatomy of white matter tracts and relation to ophthalmic examination}

We identified the optic tract (OT) and radiation (OR) using probabilistic fiber tractography which has been shown to provide improved results for mapping white matter structures (Pestilli et al. 2014; Sherbondy et al. 2008). All methods were reported in previous publications (Sherbondy et al. 2008; Ogawa et al. 2014).

Diffusion properties (Fractional Anisotropy, FA; Mean Diffusivity, MD; Radial Diffusivity, RD; Axial Diffusivity, AD) were calculated separately at 100 locations along the length of the OT and OR, these measurements hereafter are referred to as tract profiles (Yeatman et al. 2012; Rykhlevskaia et al. 2009). This visualization enables us to compare the diffusion properties of individual subjects with controls along the full path of the OT and OR.

We compared the OR tract profiles in individual AMD patients with the distribution of tract profiles in the control groups. To do so, we computed mean and standard deviation of the diffusion properties at each 1 of the 100 locations in the OT and OR. We excluded the first and last $10 \%$ of these locations leaving us with 80 measurements per white matter tract. Figure 2 shows the measurements. Statistical analyses were performed by computing a nonpaired t-test along these tract profiles as described in previous studies (Yeatman et al. 2012; Ogawa et al. 2014; Allen et al. 2015; Rykhlevskaia et al. 2009; Ajina et al. 2015; Leong et al. 2016; Gomez et al. 2015).

\section{A}
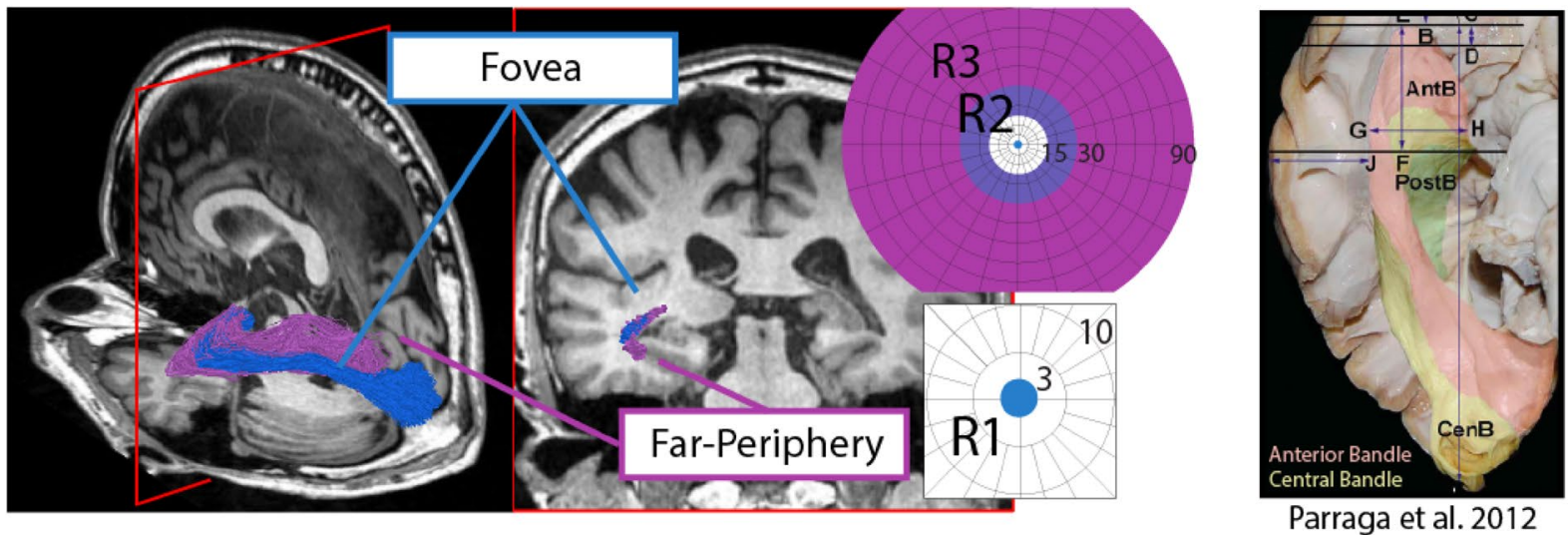

B
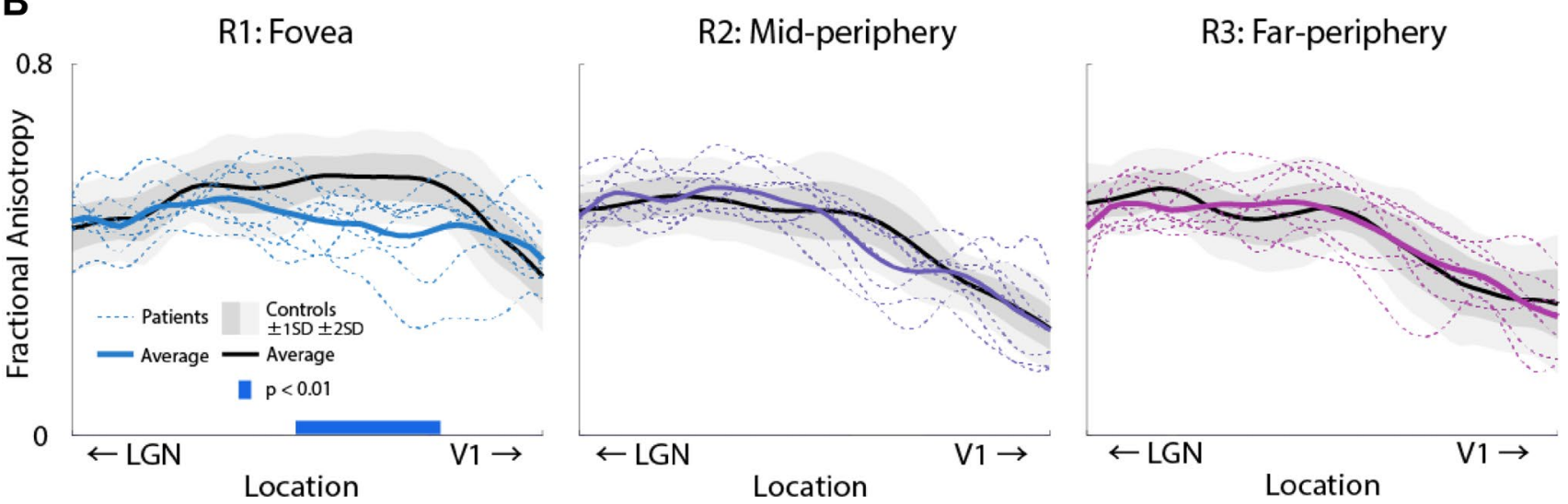

Fig. 2 White matter properties along optic radiation grouped by V1 field-map eccentricity. a We combine primary visual cortex surface topology and fascicle properties to separate white projections to fovea and periphery. Left-hand panel. The anatomical organization of the optic radiation (OR) fascicles showing spatially separated fascicles projecting to foveal and peripheral visual cortex. Right-hand panel. We show both a post-mortem anatomical segmentation depicting the known separation of the OR fascicle bundles projecting to fovea and periphery. The two bundles are generally referred to as the central bundle (yellow) and anterior bundle (salmon). Combining V1 surface topology and white matter fascicle length properties separates bun- dles projecting primarily to fovea (blue, R1) and periphery (purple, R3). b Tract profiles of white matter summary statistics. Fractional anisotropy (FA) is plotted along the length of the optic radiation for AMD patients and control participants for white matter bundles projecting to different visual field locations. Individual patients, dashed lines. Group averages, thick lines, black control group. Shaded areas \pm 1 and 2 standard deviations (SD) in the control group. Locations of significant difference, $\mathrm{p}<0.01$, between patient and control groups are highlighted by a solid blue bar at the bottom of the plot. We found that only about $21 \%$ of the white matter voxels were shared between the fovea (R1), mid- and far-periphery (R2 and R3, respectively) 


\section{Visual field maps and white matter}

In addition to identifying the full anatomy of the OT and $\mathrm{OR}$, we also identified the relation between the terminations of the OR fascicles and visual field eccentricity in V1. It is established that human V1 is organized into a retinotopicconserving map structure (Wandell et al. 2007; Wandell and Smirnakis 2010; Wandell and Winawer 2011, 2015). This means that nearby locations in the visual environment map to nearby locations in the retina and in V1.

It has been shown that the retinotopic organization of V1 can be mapped using anatomical methods (Benson et al. 2012). We used the atlas by Benson and colleagues to identify three regions within V1 of each participant in the study. V1 was subdivided into three regions (R) depending on their putative visual field eccentricity mapping. The three regions mapped from central fovea to far periphery with predicted eccentricity values are divided as follows: $\mathrm{R} 1,0^{\circ}-3^{\circ} ; \mathrm{R} 2,15^{\circ}-30^{\circ} ; \mathrm{R} 3,30^{\circ}-90^{\circ}$. We used these three $\mathrm{V} 1$ regions to subdivide the white matter fascicles of the OR into three groups with fascicles each terminating into different $\mathrm{V} 1$ regions $\mathrm{R} 1, \mathrm{R} 2$ and $\mathrm{R} 3$. This was possible because both the length and termination of white matter fascicles differed depending on their projection to V1, see Fig. 3a. Given the resolution of the dMRI data, the spatial uncertainty of tractography to accurately map fascicles within individual voxels and the uncertainty added by the mapping between anatomical atlas to each individual brain, the effective eccentricity mapping between R1, R2 and R3 might be slightly different than the nominal one. For this reason, we refer to R1 as Fovea, R2 as Mid-periphery and R3 as Far-periphery regions.

\section{Visual acuity measurements and white matter}

We investigated the relation between white matter properties in the OR and behavioral measurement of visual acuity. To do so, we used a standard Landolt-C ophthalmologic test to measure visual acuity at either fovea or at the preferred retinal locus of each individual patient-no measurements were taken in control subjects. We correlated the visual acuity measurement with those in white matter properties in each participant. White matter measures (FA, MD, AD and RD) were averaged across both for this analysis, Fig. 3 shows the results.

\section{Results}

\section{Visual white matter and retinal damage}

Goldmann perimetry measurements revealed absolute scotomas in the most central retinal locations of AMD patients, with improved retinal function at higher eccentricity (Fig. 1). We measured the anatomical shape and position of the optic tract (OT) and optic radiation (OR) in all patients and control
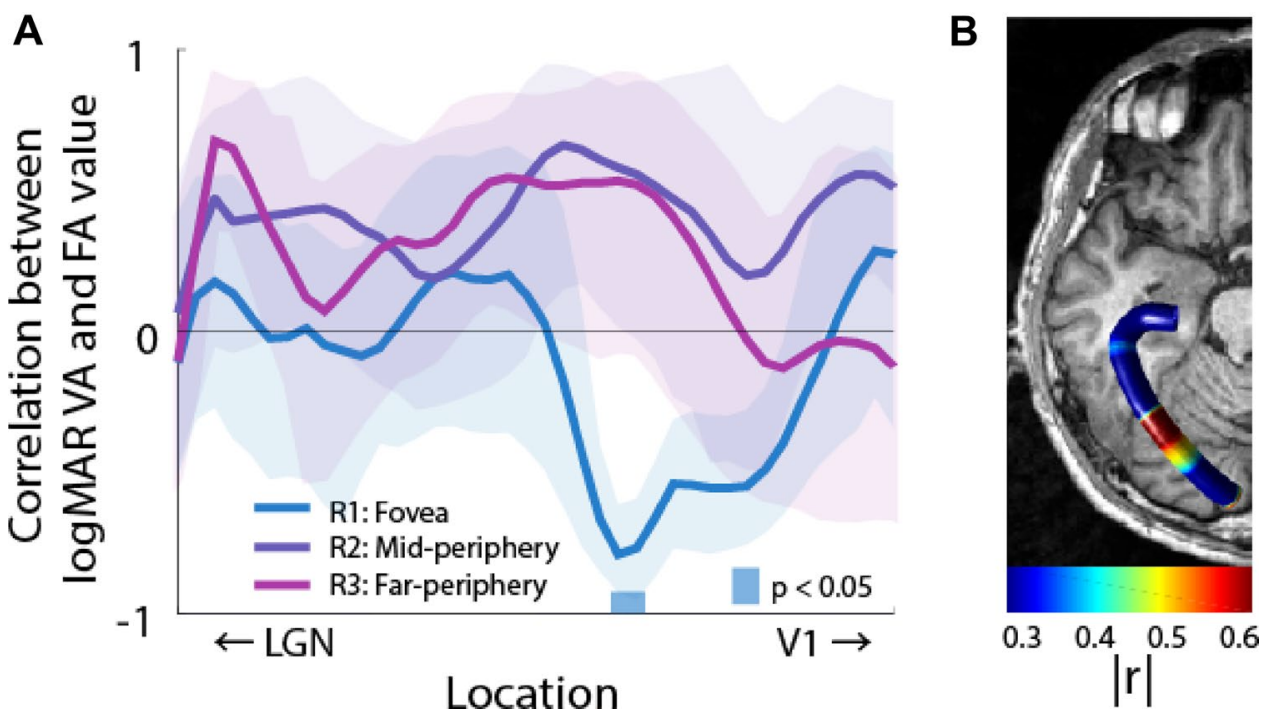

Fig. 3 Correlation analysis between visual acuity and white matter damage. a FA values along the OR correlated with participants' logMAR visual acuity at the location of white matter damage. Shaded areas show $80 \%$ confidence interval. Light blue band above the abscissa shows location of statistically significant correlation between $\log$ MAR visual acuity and FA. Darker blue band below abscissa reports locations of significantly different FA values between AMD and control subjects (see Fig. 2b for reference). b Anatomical position of the results. Average tract profile displaying the correlation between behavioral acuity and FA along the anatomy of the OR. All data display estimates for the foveal region (R1). Two nodes in the core white matter of the OR were found to be statistically significant with $p<0.05$ 
subjects (Fig. 2 and Supplementary Figs. 1-2). We measured the white matter tissue along the OT and OR using tract profiles (Yeatman et al. 2012; Ogawa et al. 2014; Allen et al. 2015; Ajina et al. 2015; Duan et al. 2015; Gomez et al. 2015; Leong et al. 2016; Libero et al. 2016) and the tensor model (Basser and Jones 2002; Basser and Pierpaoli 1998). Supplementary Fig. 1a shows mean and individual FA tract profiles of AMD patients (red) as well as the mean across control subjects (black) in the OT. Measurements show no statistically significant difference in FA between AMD patients and controls in the optic tract (Supplementary Fig. 1a). This is in contrast with a statistically significant difference in FA in the OR (Supplementary Fig. 1b, orange bar; $p<0.01$ ).

Because of the stereotyped location of retinal damage in the fovea of the AMD patients, we predicted that the white matter tissue of optic radiation (OR) would be affected primarily in fascicles projecting to the central visual field. To test this prediction, we subdivided the OR into three branches projecting to foveal (R1), mid-periphery (R2) and far-periphery (R3) locations (Fig. 2). To do so, we used a recent automatic retinotopic mapping methods (Benson et al. 2012, 2014) and the known anatomical subdivision of the OR (Fig. 2a, b) to separate the white matter projecting to the three eccentricity ranges of primary visual cortex (V1; see "Methods" for additional details).

Fractional anisotropy (FA), mean diffusivity (MD), axial diffusivity (AD) and radial diffusivity (RD) were measured for each participant along the OR. Figure $2 b$ shows mean and individual FA tract profiles of AMD patients (red), as well as the mean across control participants (black). OR FA measures differed between patients and controls. Mean FA values within the central portion of the OR (core white matter) were significantly different between patients and control subjects ( $t$ test, $\left.p<10^{-34}\right)$. Furthermore, FA was lower in AMD patients at a specific location along the optic radiation (Fig. 2b, left-hand panel; $p<0.01, t$ test). Supplementary Fig. 2a-c shows the results for MD, AD and RD. We went beyond the tensor model to show that the measured effect is robust to the choice of model and parameters used for characterizing the diffusion signal.

We further tested the specificity of the effect in the OR to demonstrate that the result is not driven by other differences in the groups of controls and patients. To do so, we mapped 11 major human white matter tracts, namely the inferior and superior longitudinal fasciculi (ILF and SLF, respectively), the arcuate fasciculus, the uncinate fasciculus, the inferior fronto occipital fasciculus (IFOF), cingulum hippocampus and cingulate, the callosum forceps major and minor, corticospinal tract and the thalamic radiation (Caiafa and Pestilli 2017; Pestilli et al. 2012, 2014). Supplementary Fig. 2d reports the tract profiles for all these tracts (average between the two hemispheres, except forceps major and minor). We compared the FA values in each of these tracts between control and patients groups. A total of 440 (40 nodes in 11 tracts) were compared; no statistical difference was found in the majority of the white matter in these tracts, with the exception of 15 nodes ( $p<0.01$; five in the cingulum, five in the IFOF, two in the callosum forceps major and three in the callosum forceps minor). The small number of nodes passing significance is expected given the large number of comparisons (440). The lack of reliable differences in FA in the major white matter tracts suggests that the strong differences in FA in the OR might be due to the relation between retinal damage and the visual white matter, and not by generalized brain differences between the two groups. To further investigate this, next we compared whether a relation exists between individual subjects visual acuity (VA) and the FA in the OR.

\section{Visual white matter and behavior: fractional anisotropy of white matter projecting to fovea predicts visual acuity}

We established the association between patients' logMAR visual acuity (hereafter only referred to as "visual acuity") and white matter properties of the OR. We predicted that FA values in individual patients would be associated with visual acuity; such that the worse the visual acuity the lower FA. We tested this prediction by measuring best corrected visual acuity in each patient using a standard Landolt-C ophthalmologic test (see "Methods").

In sum, these results show a fine-scale anatomical mapping between location of putative neurodegeneration, retinal scotoma and behavioral impairment. The result is consistent with previous reports of correlation between FA and visual acuity in other types of visual disease, such as retinitis pigmentosa (Ohno et al. 2015), and suggests that the magnitude of FA along the optic radiation may be an important predictor of visual impairment in AMD patients.

\section{Visual white matter and disease diagnosis: the tissue properties of the optic radiation predict subject class membership}

Diagnosing visual disease from brain tissue properties and investigating the relation between brain and eye disease is critical to advancing a comprehensive understanding of the visual disease (Wandell and Le 2017). Here, we were interested in understanding the degree to which the white matter properties of the optic radiation to predict the subject class membership. For this reason, we performed two additional analyses. First, we used the measured properties of white matter tissue microstructure (FA) at fifty locations along the optic radiation and performed a logistic regression analysis (Hastie et al. 2013; Green and Swets 1988) to separate the subjects into two groups, patients or controls 


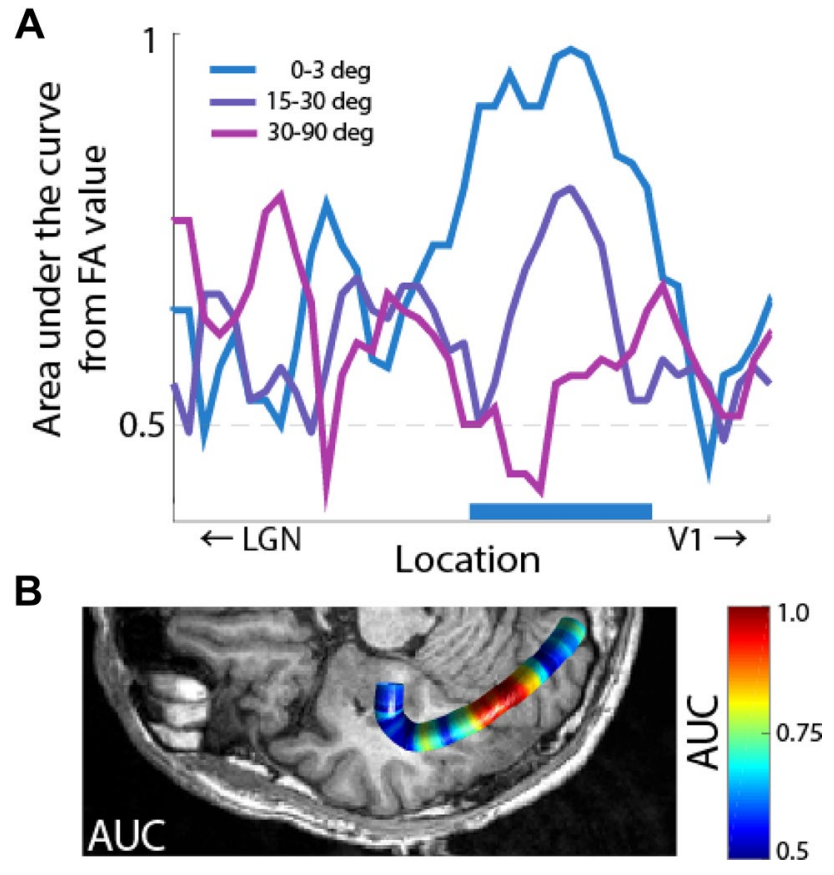

Fig. 4 White matter along the optic radiation predicts subject class membership. a Horizontal axis. Spatial position along the optic radiation. Vertical axis. Area under the curve (AUC) of a logistic analysis in which FA values from different locations in the optic radiation were used to classify subjects as patients and controls. A value of 0.5 indicates no classification. A value of 1 indicates $100 \%$ classification performance; each subject is correctly classified in the correct category using FA values at that location of the OR. b Anatomical localization of the measurements in a. Anatomical localization of the AUC values. AUC is plotted as function of location along the OR

(Fig. 4a). Logistic regression measures the area under the curve (AUC) to indicate classification performance. Our analysis compared two models: (1) one model assumed that patients and controls groups were different and (2) another in which they were assumed to not be different. Higher AUC values indicated that the model separating subjects groups (model 1) has a higher statistical power than other one model 2 (Hastie et al. 2013; Green and Swets 1988). Results demonstrated that FA in specific location along the OR allowed distinguishing between the two patient groups (Fig. 3b). Furthermore, FA was more predictive of subject group for fascicles projecting to foveal V1 (max AUC R1: 0.979, R2: 0.802, R3: 0.791). To establish whether all segments of the $\mathrm{OR}$ (R1, R2 or R3) predicted class membership or just the segment projecting to the location of retinal damage (R1), we used a bootstrap procedure (Efron and Tibshirani 1994). We repeated the logistic-regression analysis 1000 times by resampling subjects with replacement. This allowed us to estimate the confidence intervals for each cure given the data. Results showed that only the properties of the visual white matter projecting to fovea (R1 Fig. 4a, blue) significantly predicted subjects' class membership—the $95 \%$ CI were above chance level (50\%) in the core white matter for R1 (Fig. 4a blue horizontal bar).

\section{Discussion}

We presented an advanced anatomical characterization of the relation between white matter tissue microstructure in the optic radiation and optic tract of patients affected by agerelated macular degeneration. Our analyses, to our knowledge, are the first to demonstrate a conserved anatomical relation between the location of retinal damage (scotoma) and retinotopic-specific damage to white matter fascicles within the OR (Dougherty et al. 2005; Levin et al. 2010). Additionally, we report that the extent of visual impairment (behavioral measurement of visual acuity) in AMD patients is associated to the magnitude of white matter damage within the optic radiation. Hernowo et al. (2014) recently reported a reduction in the volume of the optic radiation using voxelbased morphometry in both juvenile and age-related macular degeneration (Hernowo et al. 2014; Prins et al. 2016b). The present results build upon and extend these previous reports. In addition, our results are also consistent with a series of recent report demonstrating that the basic anatomy, network organization of the visual field maps in V1 is unaffected in individuals with macular degeneration (Baseler et al. 2010, 2011; Shao et al. 2013; Haak et al. 2016). We demonstrate that the effect of macular degeneration has very specific and anatomically localized effects on the visual white matter and that the magnitude of these effects predicts visual acuity. Importantly, we show that such effects are robust and can be measured using in vivo method and magnetic resonance imaging data with clinical quality when such data are associated with advanced anatomical characterization.

We identified both the optic tracts and chiasms in every subject and found no significant decrease in FA in either of these structures. There are at least two reasons for such small changes in pregeniculate white matter: (1) there is no effect or (2) our measurements do not provide sufficient resolution to approach these structures. The human optic tract is a small structure on the order of 2-3 mm in diameter. It is embedded in an area with complex morphology where corticospinal fluid and supportive tissue types are intermixed (Parravano et al. 1993). Our advanced dMRI measurements map brain tissue at $2 \mathrm{~mm}$ resolution. For this reason, it is likely that signals from the optic tract mixed with those of nearby tissues hiding any possible effect of AMD to the optic tract. We speculate that given the retinal damage due to AMD and our reports of associated damage to post-geniculate white matter damage to pregeniculate white matter is highly likely. Future higher resolution studies might be able to report such damage. Our results are consistent with recent reports demonstrating that the white matter within the optic radiation 
is affected by AMD (Malania et al. 2017). We go beyond these previous reports by utilizing an anatomically-informed method that allowed us to characterize the specificity of the relation between locations of retinal damage and the visual white matter.

Our results extend a series of reports demonstrating a pervasive effect of eye disease on the visual white matter (Ariel Rokem et al. 2017; Millington et al. 2014). The biological mechanisms relating eye disease and the visual white matter are not completely understood. Indeed, the white matter could be affected by eye disease in at least several ways. For example, retrograde and anterograde degeneration [such as Wallerian degeneration (Pierpaoli et al. 2001; Rotshenker 2007)] could all be factors affecting the visual white matter depending on the type of disease. The current understanding of the etiology of macular degeneration is that it might be due to abnormal neo-vascularization that causes the death of photoreceptors and it is likely confined within the retinal tissue (Mandai et al. 2017). This suggests possible mechanisms for transsynaptic effects from the photoreceptors to the brain tissue. Indeed, our current results, previous ones (Malania et al. 2017) as well as results demonstrating a reduction in cortical volume in AMD (Hernowo et al. 2014; Prins et al. 2016a, b) all provide strong convergent evidence that cell death within the retina could affect the brain tissue via transsynaptic degeneration (Prins et al. 2016a; Haak et al. 2016).

The importance of precision approaches to the study of the human brain has been recently highlighted (Dubois and Adolphs 2016). We demonstrate the importance of informed anatomical characterization, methodology and study design in clarifying primary mechanism in the human white matter (Wandell 2016; Devlin and Poldrack 2007). We also show that beside easy to give away criticisms (Thomas et al. 2014), dMRI and fiber tractography offer much to inform scientists about fundamental mechanisms of the brain. In our case, the careful combination of post-mortem anatomy, with cutting edge in vivo computational neuroanatomy methods provided convergent knowledge to design experiments helpful to characterize very specific effects of neurodegeneration in the human brain. The magnitude of the effect reported here in predicting brain and behavior effects from eye disease is enhanced by the fact that the results can be measured even at the clinical resolution of the data we used. There is much to learn about the human brain and convergent evidence from diverse measurements, models and statistical methods is paramount for discovery (Pestilli 2015, 2018; Ling et al. 2015).

\section{The visual white matter for predicting behavioral function, patient diagnosis and clinical intervention}

A large proportion of AMD cases are believed to be neurovascular in nature (Wood et al. 2000; Jager et al. 2008; Fine et al. 2000). For this reason, anti-vascular endothelial growth factor (VEGF) therapy is the most widely used form of treatment for AMD. Recently, modern intravitreal anti-VEGF therapy has improved treatment of AMD. Yet, unfortunately, not all participants respond to these therapies. A mere $40 \%$ of patients regain full visual function after intravitreal antiVEGF therapy (D. M. Brown et al. 2006; Gonzalez and Gonzalez 2011; Heier et al. 2012). To date, it is not clear why different AMD patients respond differently to this treatment. Measurements of white matter damage as a result of AMD could, in principle, be used to differentiate patients in different subgroups. The capacity to characterize different subtypes of patients given the extent of white matter damage could, in turn, improve the prediction power of therapeutic efficacy of treatments, such as intravitreal anti-VEGF therapy and novel iPS (induced pluripotent stem) cell transplant therapy (Mandai et al. 2017), for refractory retinal diseases.

A number of studies have recently reported changes in white matter microstructure along the primary visual pathways as a result of ophthalmic eye disease [see (Prins et al. 2016b; Brown et al. 2016; Wandell 2016; Rokem et al. 2017) for reviews]. Major examples of impaired white matter tissue microstructure within the primary visual pathways have shown that retinitis pigmentosa (Pan et al. 2007), Leber hereditary optic neuropathy (Barcella et al. 2010; Milesi et al. 2012; Rizzo et al. 2012; Ogawa et al. 2014), amblyopia (Allen et al. 2015; Duan et al. 2015), glaucoma (Dai et al. 2013; Engelhorn et al. 2012; Garaci et al. 2009; Lee et al. 2014; Hernowo et al. 2011) and optic neuritis (Ciccarelli et al. 2005; Sergott 2011; Li et al. 2009) can affect white matter microstructure estimated in vivo using either diffusion-weighted magnetic resonance imaging methods or volumetric analyses. The current work adds to the growing evidence of strong ties between a variety of eye disease and corresponding major effects on brain tissue. It suggests that eye diseases might cause a generalized change in brain tissue properties. Future studies will be necessary to identify the extent to which damage to brain tissue microstructure due to eye disease goes beyond effects in the early visual pathways. Clarifying the extent of damage to white matter beyond the early visual pathways will have the potential to help identify differences between groups of patients and clarify reasons for differences in the effectiveness of treatments.

Acknowledgements We thank Brian A. Wandell, Sophia VinciBooher, Brent McPherson and Bradley Caron for comments on an early version of the manuscript. Takaaki Hayashi for clinical evaluation of the patients. Ikuya Murakami for institutional support. Y.S. is supported by JSPS Grant-in-Aid for Young Scientists (B) 80570332. S.O. is supported by JSPS Grant-in-Aid for Young Scientists (B) 17K18131. H.H. is supported by JSPS Grant-in-Aid for Young Scientists 18K16939 and Charitable Trust Fund for Ophthalmic Research in Commemoration of Santen Pharmaceutical's Founder. Y.M. is supported by The Jikei University Research found and Charitable Trust Fund for Ophthalmic Research in Commemoration of Santen Pharmaceutical's Founder. 
F.P. is supported by NSF IIS-1636893, NSF BCS-1734853, NIH NIMH ULTTR001108, a Microsoft Research Award and the Indiana University Areas of Emergent Research initiative "Learning: Brains, Machines, Children.” and Pervasive Technology Institute.

Author contributions Designed the study: SO HT TN YM. Performed the experiments: SY SO HH MT AM YM. Analyzed the data: SY SO HH FP. Institutional support: KM. Wrote the paper: SY SO HH FP.

\section{Compliance with ethical standards}

Conflict of interest Authors declare no conflict.

Research involving human participants and/or animals Research was approved by the ethical committees of the Jikei University School of Medicine, Tamagawa University, and Atsugi City Hospital.

Informed consent All participants provided written informed consent to participate in the project.

Open Access This article is distributed under the terms of the Creative Commons Attribution 4.0 International License (http://creativeco mmons.org/licenses/by/4.0/), which permits unrestricted use, distribution, and reproduction in any medium, provided you give appropriate credit to the original author(s) and the source, provide a link to the Creative Commons license, and indicate if changes were made.

\section{References}

Ajina S, Pestilli F, Rokem A, Kennard C, Bridge H (2015) Human blindsight is mediated by an intact geniculo-extrastriate pathway. eLife. https://doi.org/10.7554/eLife.08935

Allen B, Spiegel DP, Thompson B, Pestilli F, Rokers B (2015) Altered white matter in early visual pathways of humans with amblyopia. Vision Res 114:48-55

Allen B, Schmitt MA, Kushner BJ, Rokers B (2018) Retinothalamic white matter abnormalities in amblyopia. Invest Ophthalmol Vis Sci 59(2):921-929

Ashburner J, John A (2009) Computational anatomy with the SPM software. Magn Reson Imaging 27(8):1163-1174

Ashburner J, John A (2012) SPM: a history. NeuroImage 62(2):791-800

Barcella V, Valeria B, Rocca MA, Stefania B-M, Jacopo M, Lisa M, Andrea F, Pierro L, Filippi M (2010) Evidence for retrochiasmatic tissue loss in leber's hereditary optic neuropathy. Human Brain Mapp 31(12):1900-1906

Baseler H, Gouws A, Crossland M, Tufail A, Rubin G, Racey C, Morland A (2010) Large-scale cortical reorganization is absent in both juvenile and age-related macular degeneration. J Vis 9(8):733-733

Baseler HA, Gouws A, Haak KV, Racey C, Crossland MD, Tufail A, Rubin GS, Cornelissen FW, Morland AB (2011) Large-scale remapping of visual cortex is absent in adult humans with macular degeneration. Nat Neurosci 14(5):649-655

Basser PJ, Jones DK (2002) Diffusion-tensor MRI: theory, experimental design and data analysis - a technical review. NMR in Biomedicine. Wiley. http://onlinelibrary.wiley.com/doi/10.1002/ nbm.783/full. Accessed 30 Oct 2016

Basser PJ, Pierpaoli C (1998) A simplified method to measure the diffusion tensor from seven MR images. Magn Resonan Med 39(6):928-934
Benson NC, Butt OH, Ritobrato D, Radoeva PD, Brainard DH, Aguirre GK (2012) The retinotopic organization of striate cortex is well predicted by surface topology. Current Biol 22(21):2081-2085

Benson NC, Butt OH, Brainard DH, Aguirre GK (2014) Correction of distortion in flattened representations of the cortical surface allows prediction of V1-V3 functional organization from anatomy. PLoS Comput Biol 10(3):e1003538

Brown DM, Kaiser PK, Michels M, Soubrane G, Heier JS, Kim RY, Sy JD, Schneider S, ANCHOR Study Group (2006) Ranibizumab versus verteporfin for neovascular age-related macular degeneration. New Engl J Med 355(14):1432-1444

Brown HDH, Woodall RL, Kitching RE, Baseler HA, Morland AB (2016) Using magnetic resonance imaging to assess visual deficits: a review. Ophthalmic Physiol Optics 36(3):240-265

Caiafa CF, Pestilli F (2017) Multidimensional encoding of brain connectomes. Sci Rep 7(1):11491

Ciccarelli O, Olga C, Toosy AT, Hickman SJ, Parker GJM, WheelerKingshott CAM, Miller DH, Thompson AJ (2005) Optic radiation changes after optic neuritis detected by tractography-based group mapping. Hum Brain Mapp 25(3):308-316

Dai H, Yin D, Hu C, Morelli JN, Su Hu, Xu Y, Xu D (2013) Wholebrain voxel-based analysis of diffusion tensor mri parameters in patients with primary open angle glaucoma and correlation with clinical glaucoma stage. Neuroradiology 55(2):233-243

Devlin JT, Poldrack RA (2007) In praise of tedious anatomy. NeuroImage 37(4):1033-1041

Dougherty RF, Ben-Shachar M, Bammer R, Brewer AA, Wandell BA (2005) Functional organization of human occipital-callosal fiber tracts. Proc Natl Acad Sci 102(20):7350-7355

Dougherty RF, Ben-Shachar M, Deutsch GK, Hernandez A, Fox GR, Wandell BA (2007) Temporal-callosal pathway diffusivity predicts phonological skills in children. Proc Natl Acad Sci 104(20):8556-8561

Duan Y, Norcia AM, Yeatman JD, Mezer A (2015) The structural properties of major white matter tracts in strabismic amblyopia. Invest Ophthalmol Vis Sci 56(9):5152-5160

Dubois J, Adolphs R (2016) Building a science of individual differences from fMRI. Trends Cogn Sci 20(6):425-443

Efron B, Tibshirani RJ (1994) An introduction to the bootstrap. CRC Press, Boca Raton

Engelhorn T, Michelson G, Waerntges S, Hempel S, El-Rafei A, Struffert T, Doerfler A (2012) A new approach to assess intracranial white matter abnormalities in glaucoma patients: changes of fractional anisotropy detected by $3 \mathrm{t}$ diffusion tensor imaging. Acad Radiol 19(4):485-488

Fine SL, Berger JW, Maguire MG, Ho AC (2000) Age-related macular degeneration. N Engl J Med 342(7):483-492

Fischl B (2012) FreeSurfer. NeuroImage 62(2):774-781

Fischl B, Rajendran N, Busa E, Augustinack J, Hinds O, Yeo BTT, Mohlberg H, Amunts K, Zilles K (2007) Cortical folding patterns and predicting cytoarchitecture. Cereb Cortex 18(8):1973-1980

Garaci FG, Francesca B, Angelica C, Milena M, Arnoldo S, Claudio C, Roberto F, Giovanni S, Carlo N (2009) Optic nerve and optic radiation neurodegeneration in patients with glaucoma: in vivo analysis with 3-T diffusion-tensor MR imaging. Radiology 252(2):496-501

Gomez J, Pestilli F, Witthoft N, Golarai G, Liberman A, Poltoratski S, Yoon J, Grill-Spector K (2015) Functionally defined white matter reveals segregated pathways in human ventral temporal cortex associated with category-specific processing. Neuron 85(1):216-227

Gonzalez C, Gonzalez C (2011) Intravitreal ranibizumab for retrofoveolar neovascular age related macular degeneration, in pseudo vitelliform and/or drusenoid pigment epithelium detachment shape. Acta Ophthalmol 89:0-0 
Green DM, Swets JA (1988) Signal detection theory and psychophysics. Peninsula Pub, Baileys Harbor

Haak KV, Morland AB, Rubin GS, Cornelissen FW (2016a) Preserved retinotopic brain connectivity in macular degeneration. Ophthalmic Physiol Opt 36(3):335-343

Hastie T, Tibshirani R, Friedman J (2013) The elements of statistical learning: data mining, inference, and prediction. Springer, New York

Heier JS, Brown DM, Victor C, Jean-Francois K, Kaiser PK, Nguyen QD, Kirchhof B et al (2012) Intravitreal Aflibercept (VEGF trapeye) in wet age-related macular degeneration. Ophthalmology 119(12):2537-2548

Hernowo AT, Boucard CC, Jansonius NM, Hooymans JMM, Cornelissen FW (2011) Automated morphometry of the visual pathway in primary open-angle glaucoma. Invest Ophthalmol Vis Sci 52(5):2758-2766

Hernowo AT, Prins D, Baseler HA, Plank T, Gouws AD, Johanna MM, Hooymans AB, Morland MW, Greenlee, Cornelissen FW (2014) Morphometric analyses of the visual pathways in macular degeneration. Cortex 56:99-110

Hinds OP, Niranjini R, Polimeni JR, Augustinack JC, Graham W, Wald LL, Rosas HD, Andreas P, Schwartz EL, Bruce F (2008) Accurate prediction of $\mathrm{v} 1$ location from cortical folds in a surface coordinate system. NeuroImage 39(4):1585-1599

Jager RD, Mieler WF, Miller JW (2008) Age-related macular degeneration. N Engl J Med 358(24):2606-2617

Jbabdi S, Saad J, Sotiropoulos SN, Haber SN, Van Essen DC, Behrens TE (2015) Measuring macroscopic brain connections in Vivo. Nat Neurosci 18(11):1546-1555

Krüger FM, Kemp H, Sørensen TL (2013) Four-year treatment results of neovascular age-related macular degeneration with ranibizumab and causes for discontinuation of treatment. Am J Ophthalmol 155(1):89-95.e3

Lee JY, Jeong HJ, Lee JH, Kim YJ, Kim EY, Kim YY, Taekhyun R, Zang-Hee C, Young-Bo K (2014) An investigation of lateral geniculate nucleus volume in patients with primary open-angle glaucoma using $7 \mathrm{~T}$ magnetic resonance imaging. Investig Opthalmol Vis Sci 55(6):3468

Leong JK, Pestilli F, Wu CC, Samanez-Larkin GR, Knutson B (2016) White-matter tract connecting anterior insula to nucleus accumbens correlates with reduced preference for positively skewed Gambles. Neuron 89(1):63-69

Levin N, Netta L, Dumoulin SO, Jonathan W, Dougherty RF, Wandell BA (2010) Cortical maps and white matter tracts following long period of visual deprivation and retinal image restoration. Neuron 65(1):21-31

Li M, Li J, He HG, Lv B, Li WJ, Wang ZC, Xian JF (2009) Detecting optic nerve changes after optic neuritis with diffusion tensor MR imaging. NeuroImage 47:S127

Libero LE, Wesley K, Burge HD, Deshpande F, Pestilli, Kana RK (2016) White matter diffusion of major fiber tracts implicated in autism spectrum disorder. Brain Connectivity 6(9):691-699

Ling S, Jehee JFM, Pestilli F (2015) A review of the mechanisms by which attentional feedback shapes visual selectivity. Brain Struct Funct 220(3):1237-1250

Malania M, Konrad J, Jägle H, Werner JS, Greenlee MW (2017) Compromised integrity of central visual pathways in patients with macular degeneration. Invest Ophthalmol Vis Sci 58(7):2939-2947

Mandai M, Watanabe A, Kurimoto Y, Hirami Y, Morinaga C, Daimon T, Fujihara M et al (2017) Autologous induced stem-cellderived retinal cells for macular degeneration. N Engl J Med 376(11):1038-1046

Mayda A, Yoshita M, DeCarli C (2010) White Matter Hyperintensities in Aging and Dementia. In: Imaging the Aging Brain. Oxford University Press, New York. https://doi.org/10.1093/acprof:oso/97801 95328875.003.0017
Milesi J, Jacopo M, Rocca MA, Stefania B-M, Melissa P, Elisabetta P, Andrea F, Giancarlo C, Massimo F (2012) Patterns of white matter diffusivity abnormalities in Leber's hereditary optic neuropathy: a tract-based spatial statistics study. J Neurol 259(9):1801-1807

Millington RS, Ajina S, Bridge H (2014) Novel brain imaging approaches to understand acquired and congenital neuro-ophthalmological conditions. Curr Opin Neurol 27(1):92-97

Ogawa S, Takemura H, Horiguchi H, Terao M, Haji T, Pestilli F, Yeatman JD, Tsuneoka H, Wandell BA, Masuda Y (2014) White matter consequences of retinal receptor and ganglion cell damage. Invest Ophthalmol Vis Sci 55(10):6976-6986

Ohno N, Murai H, Suzuki Y, Kiyosawa M, Tokumaru AM, Ishii K, Ohno-Matsui K (2015) Alteration of the optic radiations using diffusion-tensor MRI in patients with retinitis pigmentosa. Br J Ophthalmol 99(8):1051-1054

Pan WJ, Wu G, Li CX, Lin F, Sun J, Lei H (2007) Progressive atrophy in the optic pathway and visual cortex of early blind chinese adults: a voxel-based morphometry magnetic resonance imaging study. NeuroImage 37(1):212-220

Parravano JG, Toledo A, Kucharczyk W (1993) Dimensions of the optic nerves, chiasm, and tracts: MR quantitative comparison between patients with optic atrophy and normals. J Comput Assist Tomogr 17(5):688-690

Pestilli F (2015) Test-retest measurements and digital validation for in vivo neuroscience. Sci Data 2(January): 140057

Pestilli F (2018) Human white matter and knowledge representation. PLoS Biol 16(4):e2005758

Pestilli F, Franco P, Yeatman JD, Ariel R, Kay KN, Wandell BA (2014) Evaluation and statistical inference for human connectomes. Nat Methods 11(10):1058-1063

Pierpaoli C, Barnett A, Pajevic S, Chen R, Penix LR, Virta A, Basser $P$ (2001) Water diffusion changes in wallerian degeneration and their dependence on white matter architecture. NeuroImage 13(6 Pt 1):1174-1185

Prins D, Hanekamp S, Frans WC (2016a) Structural brain mri studies in eye diseases: are they clinically relevant? A review of current findings. Acta Ophthalmol 94(2):113-121

Prins D, Plank T, Baseler HA, Gouws AD, Beer A, Morland AB, Greenlee MW, Cornelissen FW (2016b) Surface-based analyses of anatomical properties of the visual cortex in macular degeneration. PloS One 11(1):e0146684

Ratnapriya R, Chew EY (2013) Age-related macular degenerationclinical review and genetics update. Clin Genet 84(2):160-166

Rizzo G, Giovanni R, Tozer KR, Caterina T, David M, Claudia T, Emil M et al (2012) Secondary post-geniculate involvement in Leber's hereditary optic neuropathy. PloS One 7(11):e50230

Rokem A, Yeatman JD, Pestilli F, Kay KN, Mezer A, van der Walt S, Wandell BA (2015) Evaluating the accuracy of diffusion mri models in white matter. PloS One 10(4):e0123272

Rokem A, Takemura H, Bock AS, Scherf KS, Behrmann M, Wandell BA, Fine I, Bridge H, Pestilli F (2017) The visual white matter: The application of diffusion MRI and fiber tractography to vision science. J Vis 17(2):4

Rotshenker S (2007) Wallerian degeneration. In: Schmidt RF, Willis WD (eds) Encyclopedia of Pain. Springer, Berlin, pp 26592662. https://doi.org/10.1007/978-3-540-29805-2_4855

Rykhlevskaia EI, Sherbondy A, Wandell BA, Dougherty RF (2009) Differential growth of major white matter tracts in healthy children: a longitudinal study using DTI tractography. NeuroImage 47:S128

Sergott RC (2011) Directional diffusivity changes in the optic nerve and optic radiation in optic neuritis. Yearbook Ophthalmol 2011:196

Shao Y, Keliris GA, Papanikolaou A, Fischer MD, Zobor D, Jägle H, Logothetis NK, Smirnakis SM (2013) Visual cortex organisation 
in a macaque monkey with macular degeneration. Eur J Neurosci 38(10):3456-3464

Sherbondy AJ, Dougherty RF, Ben-Shachar M, Napel S, Wandell BA (2008) ConTrack: finding the most likely pathways between brain regions using diffusion tractography. $\mathrm{J}$ Vis $8(9): 15.1-16$

Thomas C, Moya L, Avidan G, Humphreys K, Jung KJ, Peterson MA, Behrmann M (2008) Reduction in white matter connectivity, revealed by diffusion tensor imaging, may account for age-related changes in face perception. J Cogn Neurosci 20(2):268-284

Thomas C, Ye FQ, Irfanoglu MO, Modi P, Saleem KS, Leopold DA, Pierpaoli C (2014) Anatomical accuracy of brain connections derived from diffusion MRI tractography is inherently limited. Proc Natl Acad Sci 111(46):16574-16579

Thomason ME, Thompson PM (2011) Diffusion imaging, white matter, and psychopathology. Annu Rev Clin Psychol 7(1):63-85

Wandell BA (2016) Clarifying human white matter. Annu Rev Neurosci 39:103-128

Wandell BA, Le RK (2017) Diagnosing the neural circuitry of reading. Neuron 96(2):298-311

Wandell BA, Smirnakis SM (2010) Plasticity and stability of visual field maps in adult primary visual cortex. Nat Rev Neurosci. https ://doi.org/10.1038/nrn2741

Wandell BA, Winawer J (2011) Imaging retinotopic maps in the human brain. Vis Res 51(7):718-737

Wandell BA, Winawer J (2015) Computational neuroimaging and population receptive fields. Trends Cogn Sci 19(6):349-357

Wandell BA, Serge O, Dumoulin, Brewer AA (2007) Visual field maps in human cortex. Neuron 56(2):366-383
Wang Y, Yang W, West JD, Flashman LA, Heather A, Wishart LA, Rabin P, Nadia et al (2010) Diffusion tensor MRI in preclinical Alzheimer's disease: decreased fractional anisotropy in parahippocampal white matter in MCI and older adults with cognitive complaints. Alzheimer's Dement 6(4):S37-S38

Wang Y, West JD, Flashman LA, Wishart HA, Santulli RB, Rabin LA, Saykin AJ, Pare N, Arfanakis K (2012) Selective changes in white matter integrity in MCI and older adults with cognitive complaints. Biochem Biophys Acta 1822(3):423-430

Westin CF, Peled S, Gudbjartsson H, Kikinis R, Jolesz FA et al (1997) Geometrical diffusion measures for mri from tensor basis analysis. Proc ISMRM 97:1742

Westin CF, Maier SE, Mamata H, Nabavi A, Jolesz FA, Kikinis R (2002) Processing and visualization for diffusion tensor MRI. Med Image Anal 6(2):93-108

Wong WL, Su X, Li X, Cheung CM, Klein R, Cheng CY, Wong TY (2014) Global prevalence of age-related macular degeneration and disease burden projection for 2020 and 2040: a systematic review and meta-analysis. Lancet Global Health 2(2):e106-e116

Wood AJJ, Stuart L, Fine JW, Berger MG, Maguire, Ho AC (2000) Age-related macular degeneration. N Engl J Med 342(7):483-492

Yeatman JD, Dougherty RF, Myall NJ, Wandell BA, Feldman HM (2012) Tract profiles of white matter properties: automating fibertract quantification. PloS One 7(11):e49790

Yeatman JD, Brian A, Wandell, Mezer AA (2014) Lifespan maturation and degeneration of human brain white matter. Nat Commun $5: 4932$ 